\title{
Rate 2 Quasi-Orthogonal Space Time Block Codes using Parallel Interference Cancellation
}

\author{
Nicholas S. J. Pau, Desmond P. Taylor, Philippa A. Martin \\ Electrical and Computer Engineering Department, \\ University of Canterbury, Private Bag 4800, Christchurch, NEW ZEALAND. \\ Email: \{nsp14, taylor, p.martin\}@elec.canterbury.ac.nz.
}

\begin{abstract}
In this paper, we focus on an advanced space-time system using a rate 2 quasi-orthogonal space-time block code which enables us to achieve very good performance for an overall throughput of up to $4.3 \mathrm{bits} / \mathrm{s} / \mathrm{Hz}$. This is done through the use of a bit-mapped coded modulation structure using short low density parity check component codes. At the receiver, parallel interference cancellation (PIC) and belief propagation (BP) decoding are employed. Iterative decoding is performed between the PIC and BP decoding stages. Given a fixed total number of decoding iterations, we investigate the frequency of PIC updates required to achieve good performance.
\end{abstract}

\section{INTRODUCTION}

Alamouti introduced a simple space-time block code (STBC) for two transmit antennas in [1]. This was generalized to an abitrary number of transmit antennas in [2]. All these STBCs are orthogonal STBCs (OSTBCs) and have rates of one or less. They may be maximum-likelihood (ML) decoded using simple linear processing.

One goal of space time coding is to increase channel capacity. Therefore, space-time schemes which provide rates greater than one have also been designed, including quasiorthogonal STBCs (QOSTBCs) [3], [4], [5], the double spacetime transmit diversity (DSTTD) scheme [6] and the Bell Labs Layered Space-Time (BLAST) Architecture [7]. The DSTTD scheme is essentially a QOSTBC which combines the Alamouti OSTBC [1] with BLAST [7]. All these schemes employ either joint detection [3] or ordered successive interference cancellation (SIC) [4], [5], [6], [7] and have detection complexities that increase exponentially with the number of interferers.

In this paper, we design a MIMO system which achieves high throughput with good performance using a low complexity detection scheme. To increase throughput, we use the QOSTBC (DSTTD scheme) of [6] which has rate 2. Good performance is achieved using bit-mapped coded modulation (BMCM) employing low density parity-check (LDPC) codes as component codes. Unlike bit-interleaved coded modulation (BICM) [8], BMCM does not require interleaving and hence has shorter delays. We employ iterative parallel interference cancellation (PIC) [9], [10] at the receiver to minimize the effects of interference during symbol estimation. The PIC scheme has lower detection complexity than joint detection or ordered SIC, especially with an increasing number of interferers. The LDPC component codes may be decoded using the belief propagation (BP) algorithm, which is considered to have low decoding complexity. The BMCM structure allows the BP decoding of each component code to be performed in parallel, which further reduces delays.

The proposed receiver structure uses two types of iterative processes. In the first iterative process, symbol estimates are demodulated and passed to parallel BP decoders. The decoded bits are re-modulated and the resulting symbols are used to update the PIC process in the following iteration. The idea of iterating between a PIC detector and parallel forward error-correction decoders was considered in [10] for a diagonal-BLAST system. The second iterative process is performed internally by the BP decoders. We investigate the ratio of PIC updates to $\mathrm{BP}$ iterations required to produce good performance, for a fixed maximum number of $\mathrm{BP}$ iterations, across all PIC updates.

In Section II, we describe the proposed BMCM-STBC system structure and the channel model used. Section III describes the iterative detection and decoding processes. Simulation results are presented in Section IV and conclusions are drawn in Section V.

\section{PRoposed System}

We consider a MIMO channel with $n_{T}$ transmit and $n_{R}$ receive antennas. We assume a quasi-static flat Rayleigh fading channel model. The encoding process is described next, followed by a description of the channel model.

\section{A. Encoding}

The proposed encoder structure is shown in Fig. 1. We consider a $2^{M}$-ary quadrature amplitude modulation (QAM) constellation. The input data stream is demultiplexed into $M$ data substreams $\left\{\boldsymbol{B}^{m}\right\}_{m=1}^{M}$. The $m^{t h}$ data substream has length $K_{m}$. Each substream is encoded using an LDPC code to

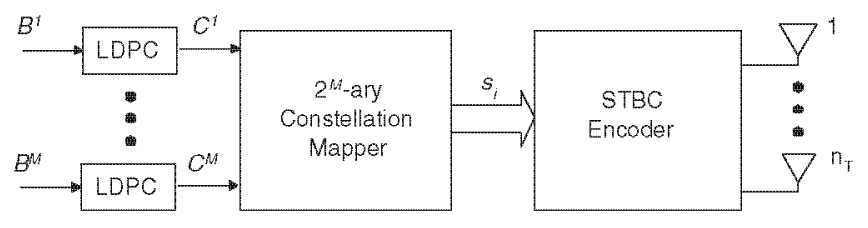

Fig. 1. Encoder block diagram. 
obtain the set of $M$ length- $N$ codewords $\left\{C^{m}\right\}_{m=1}^{M}$, where the $i^{t h}$ encoded bit in $C^{m}$ is denoted $C_{i}^{m}$. Note that each substream can be encoded using LDPC codes with different values of $K_{m}$ as long as all $M$ codes produce codewords of the same length, $N$. This requires careful choice of code rates for each substream. The overall rate of the LDPC codes is

$$
R_{l d p c}=\frac{\sum_{m=1}^{M} K_{m}}{M N} .
$$

The encoding process can be written as $\boldsymbol{C}^{m}=\boldsymbol{B}^{m} \boldsymbol{G}^{m}$, where $\boldsymbol{G}^{m}$ is the $K_{m}$-by- $N$ generator matrix of the $m^{\text {th }}$ LDPC component code. The $i^{t h}$ bits from all $M$ codewords collectively select the $i^{\text {th }} 2^{M}$-ary QAM constellation point $\boldsymbol{s}_{i}$.

A conventional rate 1 QOSTBC [3] is given by

$$
\zeta_{44}=\left[\begin{array}{cccc}
s_{4} & -s_{3}^{*} & -s_{2}^{*} & s_{1} \\
-s_{3} & -s_{4}^{*} & s_{1}^{*} & s_{2} \\
-s_{2} & s_{1}^{*} & -s_{4}^{*} & s_{3} \\
s_{1} & s_{2}^{*} & s_{3}^{*} & s_{4}
\end{array}\right],
$$

where each row is transmitted from a different antenna and each column is transmitted in a different time slot. This QOSTBC transmits four new constellation points $\left(s_{1}, s_{2}, s_{3}, s_{4}\right)$ in each block of $L=4$ time slots using four transmit antennas. The rate of a STBC is defined as

$$
R_{s t b c}=\frac{\text { number of new symbols transmitted }}{\text { number of time slots used, } L} .
$$

Therefore, the QOSTBC of (2) has $R_{s t b c}=1$. We shall refer to this STBC as the Rate 1 QOSTBC.

Here we consider the DSTTD scheme of [6] which can be described by

$$
\zeta_{42}=\left[\begin{array}{cc}
-s_{2}^{*} & s_{1} \\
s_{1}^{*} & s_{2} \\
-s_{4}^{*} & s_{3} \\
s_{3}^{*} & s_{4}
\end{array}\right]
$$

where each group of four new constellation points $\left(s_{1}, s_{2}, s_{3}, s_{4}\right)$ is transmitted using $L=2$ time slots and four transmit antennas. We note that the DSTTD scheme is merely a truncated version of the Rate 1 QOSTBC in (2). If we split the transmit antennas into Group A (Tx1, Tx2) and Group B (Tx3, Tx4), we are effectively transmitting the two groups independently (as in BLAST systems), where each group uses the simple Alamouti OSTBC. Therefore, the overall scheme is quasi-orthogonal and has $R_{s t b c}=2$, so we shall refer to it as the Rate 2 QOSTBC.

\section{B. Channel Model}

We consider a quasi-static Rayleigh flat fading channel model. Let $\alpha_{p}^{q}(t)$ denote the complex fading coefficient affecting the symbol $x_{p}(t)$ at time $t$ for the subchannel between the $p^{t h}$ transmit antenna and the $q^{t h}$ receive antenna for $p=1,2, \ldots, n_{T}$ and $q=1,2, \ldots, n_{R}$. We assume independent subchannels. Due to the quasi-static assumption, we let the fading coefficients remain fixed during each STBC block (of $L$ time slots) and vary independently from one block to the next. We model the fading coefficients as zero-mean complex Gaussian random variables with variance $1 / 2$ per dimension.
We assume that we have ideal channel state information (CSI) at the receiver.

We fix the total transmitted energy across all $n_{T}$ transmit antennas to be 1 , for each time slot. In the case of $n_{T}=4$, the symbol transmitted from each antenna contains $\frac{1}{n_{T}}=1 / 4$ unit of energy. The signal at each receive antenna is a noisy superposition of the transmitted signals after undergoing quasistatic flat Rayleigh fading, and is given by

$$
r^{q}(t)=\sum_{p=1}^{n_{T}} \alpha_{p}^{q} x_{p}(t)+w^{q}(t), \quad t=1, \ldots, L
$$

where $w^{q}(t)$ is the additive white Gaussian noise (AWGN) at the $q^{t h}$ receive antenna at time $t$ for $q=1,2, \ldots, n_{R}$. The AWGN is modeled by an independent complex Gaussian random variable with zero mean and a one-dimensional noise variance defined as

$$
\sigma^{2}=\frac{N_{0}}{2}=\frac{n_{T} E_{s}}{M R_{s t b c} R_{l d p c} 10^{0.1 S N R}}
$$

where $E_{s}$ is the average energy of a constellation point and SNR is the signal-to-noise ratio per receive antenna and is given in decibels $(d B)$. The overall throughput for a system using a $2^{M}$-ary constellation is defined as

$$
\text { Throughput }=M R_{s t b c} R_{l d p c} \quad \text { bits } / \mathrm{s} / \mathrm{Hz}
$$

where $R_{s t b c}$ is the rate of the STBC defined in (3) and $R_{l d p c}$ is the overall rate of the LDPC codes defined in (1).

\section{Detection AND DECODING}

At the receiver, linear processing [1], [2] is first applied to the received signals to produce an estimate of the transmitted symbols. The Rate 1 QOSTBC takes symbols $\boldsymbol{s}_{1}, \boldsymbol{s}_{2}, \boldsymbol{s}_{3}, \boldsymbol{s}_{4}$ and transmits them using $n_{T}=4$ antennas and $L=4$ time slots. Assuming $n_{R}=1$, linear processing at the receiver produces the estimates [3]

$$
\begin{aligned}
\hat{\boldsymbol{s}}_{\mathbf{1}}= & \left(\left|\alpha_{1}\right|^{2}+\left|\alpha_{2}\right|^{2}+\left|\alpha_{3}\right|^{2}+\left|\alpha_{4}\right|^{2}\right) \boldsymbol{s}_{\mathbf{1}} \\
& +2\left(\alpha_{1} \alpha_{4}^{*}-\alpha_{2} \alpha_{3}^{*}\right) \boldsymbol{s}_{\mathbf{4}}+\text { noise } \\
\hat{\boldsymbol{s}}_{\mathbf{2}}= & \left(\left|\alpha_{1}\right|^{2}+\left|\alpha_{2}\right|^{2}+\left|\alpha_{3}\right|^{2}+\left|\alpha_{4}\right|^{2}\right) \boldsymbol{s}_{\mathbf{2}} \\
& +2\left(\alpha_{2} \alpha_{3}^{*}-\alpha_{1} \alpha_{4}^{*}\right) \boldsymbol{s}_{\mathbf{3}}+\text { noise } \\
\hat{\boldsymbol{s}}_{\mathbf{3}}= & \left(\left|\alpha_{1}\right|^{2}+\left|\alpha_{2}\right|^{2}+\left|\alpha_{3}\right|^{2}+\left|\alpha_{4}\right|^{2}\right) \boldsymbol{s}_{\mathbf{3}} \\
& +2\left(\alpha_{2} \alpha_{3}^{*}-\alpha_{1} \alpha_{4}^{*}\right) \boldsymbol{s}_{\mathbf{2}}+\text { noise } \\
\hat{\boldsymbol{s}}_{\mathbf{4}}= & \left(\left|\alpha_{1}\right|^{2}+\left|\alpha_{2}\right|^{2}+\left|\alpha_{3}\right|^{2}+\left|\alpha_{4}\right|^{2}\right) \boldsymbol{s}_{\mathbf{4}} \\
& +2\left(\alpha_{1} \alpha_{4}^{*}-\alpha_{2} \alpha_{3}^{*}\right) \boldsymbol{s}_{\mathbf{1}}+\text { noise. }
\end{aligned}
$$

The Rate 2 QOSTBC transmits the symbols $\boldsymbol{s}_{1}, \boldsymbol{s}_{2}, \boldsymbol{s}_{3}, \boldsymbol{s}_{4}$ using $n_{T}=4$ antennas and $L=2$ time slots. For $n_{R}=1$, 
linear processing produces the estimates

$$
\begin{aligned}
\hat{\boldsymbol{s}}_{\mathbf{1}}= & \left(\left|\alpha_{1}\right|^{2}+\left|\alpha_{2}\right|^{2}\right) \boldsymbol{s}_{\mathbf{1}} \\
& +\left(\alpha_{1}^{*} \alpha_{3}+\alpha_{2} \alpha_{4}^{*}\right) \boldsymbol{s}_{\mathbf{3}}+\left(\alpha_{1}^{*} \alpha_{4}-\alpha_{2} \alpha_{3}^{*}\right) \boldsymbol{s}_{\mathbf{4}}+\text { noise } \\
\hat{\boldsymbol{s}}_{\mathbf{2}}= & \left(\left|\alpha_{1}\right|^{2}+\left|\alpha_{2}\right|^{2}\right) \boldsymbol{s}_{\mathbf{2}} \\
& +\left(\alpha_{2}^{*} \alpha_{4}+\alpha_{1} \alpha_{3}^{*}\right) \boldsymbol{s}_{\mathbf{4}}+\left(\alpha_{2}^{*} \alpha_{3}-\alpha_{1} \alpha_{4}^{*}\right) \boldsymbol{s}_{\mathbf{3}}+\text { noise } \\
\hat{\boldsymbol{s}}_{\mathbf{3}}= & \left(\left|\alpha_{3}\right|^{2}+\left|\alpha_{4}\right|^{2}\right) \boldsymbol{s}_{\mathbf{3}} \\
& +\left(\alpha_{3}^{*} \alpha_{1}+\alpha_{4} \alpha_{2}^{*}\right) \boldsymbol{s}_{\mathbf{1}}+\left(\alpha_{3}^{*} \alpha_{2}-\alpha_{4} \alpha_{1}^{*}\right) \boldsymbol{s}_{\mathbf{2}}+\text { noise } \\
\hat{\boldsymbol{s}}_{\mathbf{4}}= & \left(\left|\alpha_{3}\right|^{2}+\left|\alpha_{4}\right|^{2}\right) \boldsymbol{s}_{\mathbf{4}} \\
& +\left(\alpha_{4}^{*} \alpha_{2}+\alpha_{3} \alpha_{1}^{*}\right) \boldsymbol{s}_{\mathbf{2}}+\left(\alpha_{4}^{*} \alpha_{1}-\alpha_{3} \alpha_{2}^{*}\right) \boldsymbol{s}_{\mathbf{1}}+\text { noise. }
\end{aligned}
$$

In both cases, the interference is due to the non-orthogonality of the space-time code structure. These symbol estimates are used by either the joint detection (JD) scheme of [3] or the proposed PIC scheme to produce better estimates. The two schemes are explained in sections III-A and III-B, respectively.

\section{A. Joint Detection}

The receiver structure for the JD scheme of [3] is shown in Fig. 2. From (8), it is clear that the Rate 1 QOSTBC produces estimates that depend on the transmitted symbol plus one interferer and some noise terms. The JD scheme considers all possible pairs of constellation points between the transmitted symbol and the interferer, and selects the best pair based on Euclidean distance. The improved symbol estimates are then demodulated into soft bit estimates for the $M \mathrm{BP}$ decoders. The complexity of JD increases as $\left(2^{M}\right)^{I+1}$, where $I$ is the number of interferers. For a BMCM system using 16QAM and the Rate 1 QOSTBC, we need to search through $16^{2}=256$ possible pairs of constellation points. From (9), the Rate 2 QOSTBC produces ID estimates that depend on the transmitted symbol, two interferers and some noise terms. Therefore, the Rate 2 QOSTBC requires a search through $16^{3}=4096$ possible combinations of three constellation points.

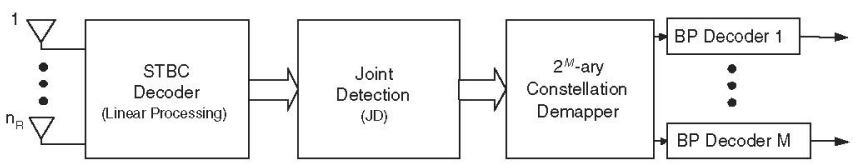

Fig. 2. Receiver structure using joint detection.

\section{B. Parallel Interference Cancellation}

The exponentially increasing complexity of the JD process as $I$ increases motivated us to find a simpler detection scheme. We employ PIC, which is widely used in multiuser detection and is considered to have lower complexity and shorter delay than ordered SIC. In [10], information is iteratively shared between the PIC block and $n_{T}$ convolutional decoders, via interleaving/de-interleaving. Here, information is iteratively exchanged between the PIC block and the $M$ LDPC decoders, via mapping/demapping. The receiver structure for our proposed iterative PIC scheme is shown in Fig. 3.

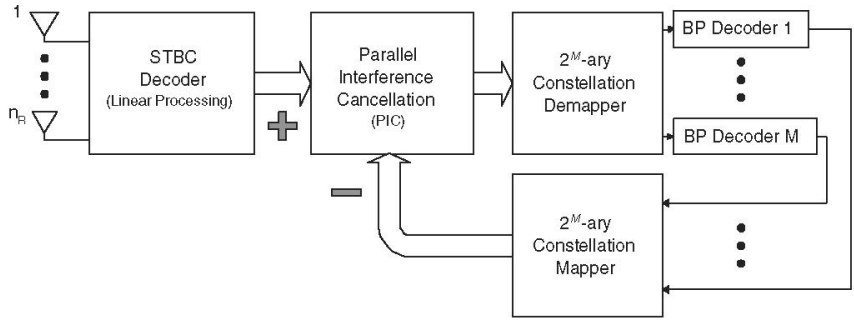

Fig. 3. Receiver structure using parallel interference cancellation.

In the first iteration, no PIC is performed after the linear processing. Instead the symbol estimates from the linear processing block are demodulated and the estimated bits are passed to the parallel LDPC decoders. The improved bit estimates from the LDPC decoders are then re-modulated to give improved estimates of the transmitted symbols, which are then used together with the CSI to cancel the interference according to (8) or (9). This generates improved symbol estimates which are demodulated and the corresponding bits passed to the parallel LDPC decoders. This iterative process is repeated until there is negligible further improvement in performance.

The LDPC decoders use the BP decoding algorithm, which requires internal iterations. This is different from the iterations between the PIC block and the LDPC decoders described previously. We use the terms BP iterations and PIC updates, respectively, to distinguish between the two iterative processes.

Unlike the SIC schemes of BLAST, no ordering is needed and the interference cancellation is done in parallel, which reduces processing complexity and delay. The complexity of each PIC update increases linearly with the number of interferers $I$ since a subtraction operation is needed for each interferer. For example, (8) shows that the Rate 1 QOSTBC requires four subtraction operations to cancel out the interference for each PIC iteration. Therefore, if 5 PIC iterations were used, the relative complexity is $(I=1)(4)(5$ iter $)=20$ operations. In the Rate 2 QOSTBC case, the relative complexity of PIC increases to $(I=2)(4)(5)=40$ operations. It is difficult to make a direct complexity comparison between the PIC scheme and the JD scheme, since the former does not require a search through combinations of constellation points.

\section{Simulation Results}

All the simulation results presented are for MIMO systems with $n_{T}=n_{R}=4$. Gray mapped 16-QAM and quadrature phase shift keying (QPSK) constellations are used. We assume perfect CSI at the receiver, but none at the transmitter. In the present work, four $(343,186)$ LDPC component codes are used. They have an overall rate of 0.54 , providing an overall throughput of approximately $4.3 \mathrm{bits} / \mathrm{s} / \mathrm{Hz}$. Each simulation point contains 100 frame errors, where each frame encompasses 172 or 2 time slots for the coded and uncoded schemes, respectively. A frame is considered to be in error if and only if any of its data bits are in error. 


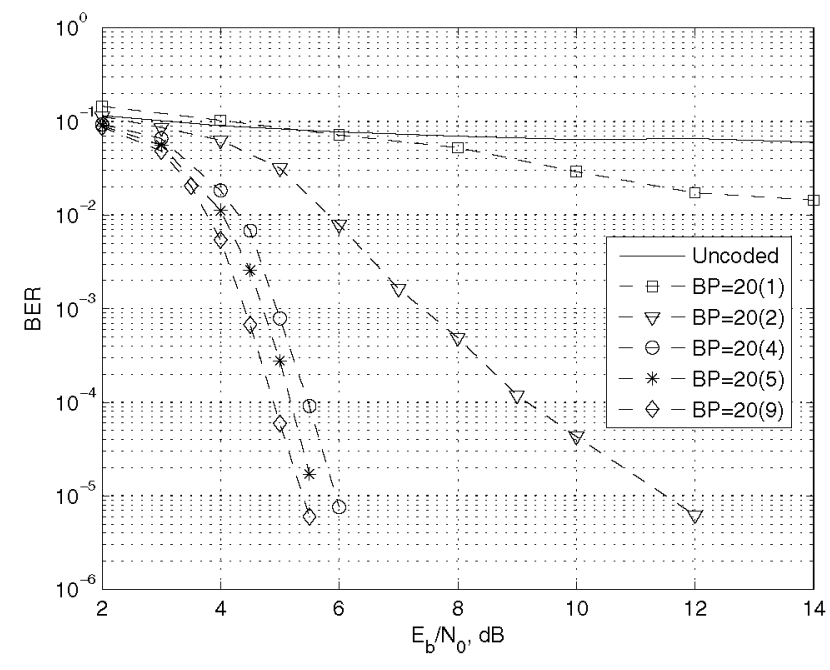

Fig. 4. BER performance of the proposed scheme using four $(343,186)$ LDPC component codes for 1,2, 4, 5 and 9 PIC updates and a maximum of $20 \mathrm{BP}$ iterations between PIC updates. Throughput is $4.3 \mathrm{bits} / \mathrm{s} / \mathrm{Hz}$.

Fig. 4 illustrates the coded bit error rate (BER) performance using the proposed 16-QAM BMCM system, LDPC component codes and the Rate 2 QOSTBC. It shows the performance after 1, 2, 4, 5 and 9 PIC updates. The BP decoders use a maximum $^{1}$ of 20 iterations between PIC updates. The notation $B P=20(5)$ indicates that a maximum of $20 \mathrm{BP}$ iterations are used between PIC updates and 5 PIC updates are used in total. This means a maximum of $120=20+5(20)$ BP iterations are used overall. The performance gain after more than 5 updates is minimal. The BER curve after 5 PIC updates is falling at almost 2 decades $/ d B$ and is not showing any signs of an error floor at $10^{-5}$. Each iteration provides a diminishing increase in coding gain.

Fig. 5 compares the uncoded BER performance of the Rate $1 / 2$ OSTBC of [2] using linear processing and the Rate 1 QOSTBC using JD [3]. In addition, we consider the coded BER performance for the Rate 1 QOSTBC using JD and PIC, and the Rate 2 QOSTBCs using PIC. The modulation schemes and LDPC codes are chosen to result in the same approximate throughput of $2 \mathrm{bits} / \mathrm{s} / \mathrm{Hz}$ in all cases. The uncoded Rate $1 / 2$ OSTBC has the worst performance in the group because it pays a big penalty for the low STBC rate, which resulted from the use of 16-QAM and no LDPC codes. It attains a BER of $10^{-4}$ at $13.2 d B$. The Rate 1 QOSTBC using JD reaches the same BER at $9.7 d B$, a gain of $3.5 d B$ over the OSTBC. This is because the Rate 1 QOSTBC has a higher rate and is able to use a smaller constellation (QPSK), which has a larger minimum Euclidean distance between constellation points.

We now compare the performance in Fig. 5 of the JD and PIC schemes when used with the Rate 1 QOSTBC, 16-QAM and the BMCM-LDPC coded scheme. When JD is used, the

\footnotetext{
${ }^{1}$ The BP algorithm uses a stopping criterion which will terminate BP decoding before 20 iterations if a codeword is found.
}

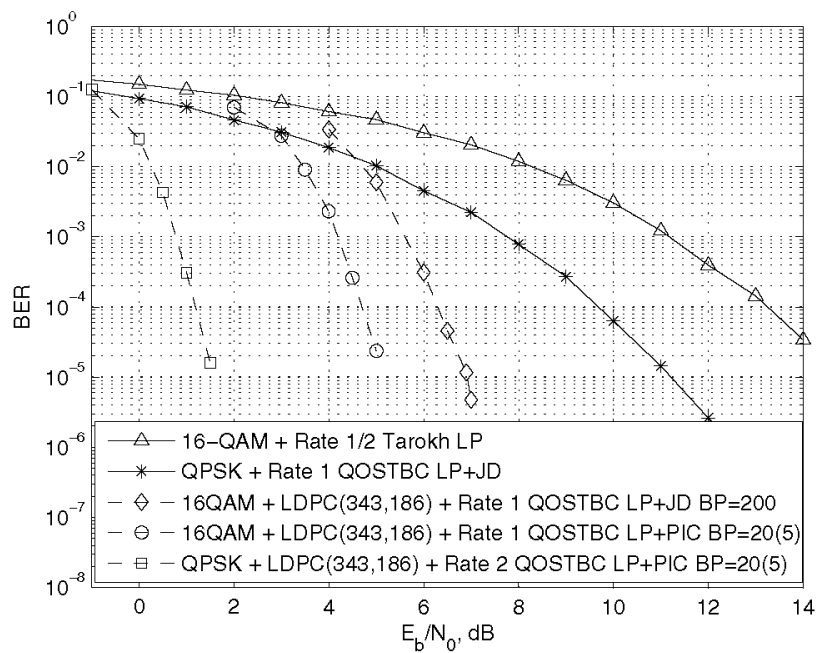

Fig. 5. BER performance comparison of coded and uncoded systems for the same approximate throughput of $2 \mathrm{bits} / \mathrm{s} / \mathrm{Hz}$.

symbol estimates from the JD block are demapped to bits and the bit estimates are passed to the parallel BP decoders. A maximum of $200 \mathrm{BP}$ iterations are used to decode each LDPC code. Due to the high complexity of the JD detection scheme, no soft information is passed back to the JD block from the BP decoders. When PIC is used, the symbol estimates on the first iteration are passed straight to the BP decoders after linear processing. On subsequent iterations, soft information from the BP decoders is passed to the PIC block. The coded JD scheme achieves a BER of $10^{-4}$ at $6.4 \mathrm{~dB}$, a gain of $3.3 \mathrm{~dB}$ over the uncoded scheme using JD. The coded PIC scheme achieves the same BER at $4.7 d B$, a further gain of $1.7 d B$ over the coded JD scheme.

When the Rate 2 QOSTBC is used in conjunction with the same LDPC component codes and PIC, QPSK produces the required throughput. As shown in Fig. 5 this scheme achieves a BER of $10^{-4}$ at about $1.2 \mathrm{~dB}$, a gain of $3.5 \mathrm{~dB}$ over the coded Rate 1 QOSTBC using PIC. This also represents an overall gain of $12 d B$ over the uncoded Rate $1 / 2$ OSTBC.

Fig. 6 compares the frame error rate (FER) and BER performance of the 16-QAM BMCM scheme using the Rate 2 QOSTBC and $(343,186)$ LDPC component codes, providing a throughput of $4.3 \mathrm{bits} / \mathrm{s} / \mathrm{Hz}$. Performance is shown for different numbers of PIC updates and BP iterations. The maximum total number of BP iterations used is fixed at $N_{\max }=100$. In both the FER and BER cases, the performance generally improves as the number of PIC updates is increased. However, increasing the number of PIC updates beyond 9 and 19 degrades the FER and BER performances, respectively. This is because the number of allowable BP iterations between PIC updates decreases to such a level that the BP algorithm is unable to converge properly. Note also that when the number of PIC updates is too high (e.g. 49), the FER performance degrades much more significantly than the BER performance. 2327 


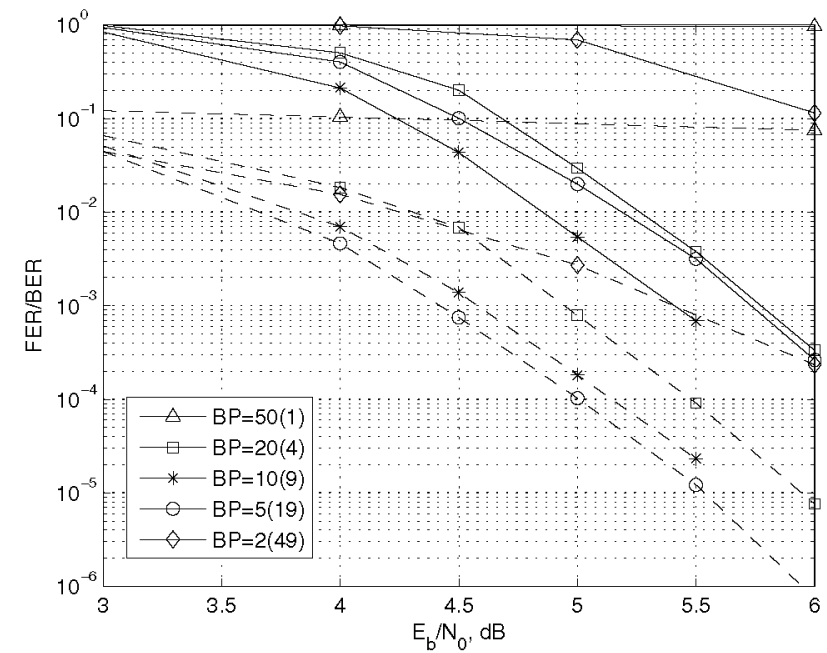

Fig. 6. FER (solid lines) and BER (dashed lines) performance of the 16-QAM BMCM scheme, using the Rate 2 QOSTBC and $(343,186)$ LDPC component codes. Performance is shown for different numbers of PIC updates when an overall maximum of $100 \mathrm{BP}$ iterations is used. Throughput is $4.3 \mathrm{bits} / \mathrm{s} / \mathrm{Hz}$.

In Fig. 7 we compare the FER and BER performance of the same $4.3 \mathrm{bits} / \mathrm{s} / \mathrm{Hz}$ scheme using different numbers of PIC updates and BP iterations, for $N_{\max }=400$. Similar trends to Fig. $6\left(N_{\max }=100\right)$ are observed. Both the FER and BER performances generally improve as the number of PIC updates increases. However, increasing the number of PIC updates beyond 9 degrades performance. The FER performance degrades significantly more than the BER performance when the number of PIC updates is further increased to 79.

Comparing Fig. 6 and Fig. 7, the curves with the best performance reach a BER of $10^{-4}$ at $5.01 \mathrm{~dB}$ and $4.76 \mathrm{~dB}$, for $N_{\max }=100(\mathrm{BP}=5(19))$ and $N_{\max }=400(\mathrm{BP}=40(9))$, respectively. Therefore, increasing $N_{\max }$ from 100 to 400 provides a small SNR gain of only $0.23 d B$. Similar gains are observed in the FER performances. To obtain good performance for a fixed $N_{\max }$, we suggest using $B P=$ $\sqrt{N_{\max }}\left(\sqrt{N_{\max }}-1\right)$ as a starting point. This corresponds to a ratio of PIC updates to $\mathrm{BP}$ iterations (between updates) of approximately 1 .

\section{CONCLUSION}

We have presented a simple new space-time coding scheme, which uses a Rate 2 QOSTBC and BMCM with LDPC component codes. The Rate 2 QOSTBC used is a hybrid of the Alamouti OSTBC and BLAST. In order to achieve low detection and decoding complexity, this scheme uses simple PIC detection together with BP decoding. Information is iteratively shared between the PIC detector and the parallel $\mathrm{BP}$ decoders to improve performance. We also investigated the effects on performance of changing the ratio of PIC updates to $\mathrm{BP}$ iterations (between updates). A ratio close to 1 is found to be a good starting point. Simulation results show we are

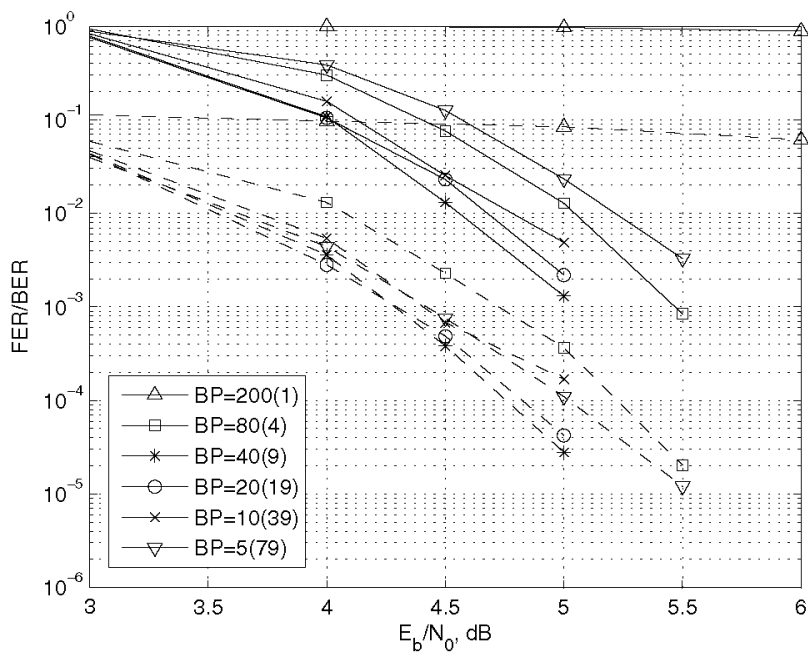

Fig. 7. FER (solid lines) and BER (dashed lines) performance of the 16-QAM BMCM scheme, using the Rate 2 QOSTBC and $(343,186)$ LDPC component codes. Performance is shown for different numbers of PIC updates when an overall maximum of $400 \mathrm{BP}$ iterations is used. Throughput is $4.3 \mathrm{bits} / \mathrm{s} / \mathrm{Hz}$.

able to achieve a throughput of $4.3 \mathrm{bits} / \mathrm{s} / \mathrm{Hz}$ using $16-\mathrm{QAM}$ and still maintain very good BER and FER performance.

\section{REFERENCES}

[1] S. Alamouti, "A simple transmit diversity technique for wireless communications," IEEE J. Select. Areas Commun., vol. 16, pp. 1451-1458, Oct 1998.

[2] V. Tarokh, H. Jafarkhani, and A. R. Calderbank, "Space-time block codes from orthogonal designs," IEEE Trans. Inform. Theory, vol. 45, pp. 1456-1467, July 1999.

[3] H. Jafarkhani, "A quasi-orthogonal space-time block code," IEEE Trans. Commun., vol. 49, pp. 1-4, Jan. 2001.

[4] M. Tao and R. S. Cheng, "Generalized layered space-time codes for high data rate wireless communications," IEEE Trans. Wireless Commun., vol. 3, pp. 1067-1075, July 2004.

[5] V. Tarokh, A. Naguib, N. Seshadri, and A. R. Calderbank, "Combined array processing and space-time coding," IEEE Trans. Inform. Theory, vol. 45, pp. 1121-1128, May 1999.

[6] E. N. Onggosanusi, A. G. Dabak, and T. A. Schmidl, "High rate spacetime block coded scheme: Performance and improvement in correlated fading channels," in Proc. WCNC, vol. 1, pp. 194-199, Mar. 2002.

[7] G. J. Foschini, "Layered space-time architecture for wireless communication is a fading environment when using multiple antennas," Bell Labs. Tech. J., vol. 1, autumn 1996.

[8] E. Zehavi, "8-PSK trellis codes for a rayleigh channel," IEEE Trans. Commun., vol. 40, pp. 873-884, May 1992.

[9] M. M. da Silva, A. M. C. Correia, J. C. Silva, and N. Souto, "Joint MIMO and parallel interference cancellation for the HSDPA," in Proc. ISSTA, pp. 424-428, Aug-Sept 2004.

[10] M. Sellathurai and S. Haykin, "A simplified diagonal BLAST architecture with iterative parallel-interference cancellation receivers," in Proc. ICC, vol. 10, pp. 3067-3071, June 2001. 